\title{
Opportunistic Error Correction for MIMO
}

\author{
Xiaoying Shao, Cornelis H. Slump \\ Department of Electrical Engineering, Mathematics and Computer Science \\ University of Twente, The Netherlands
}

\begin{abstract}
In this paper, we propose an energy-efficient scheme to reduce the power consumption of ADCs in MIMO-OFDM systems. The proposed opportunistic error correction scheme is based on resolution adaptive ADCs and fountain codes. The key idea is to transmit a fountain-encoded packet over one single subcarrier per antenna and to transmit more packets than needed for decoding. Comparing to the IEEE 802.11n standard, this approach allows around $83 \%$ of power saving in ADCs for a $2 \times 2$ system and $90 \%$ for a $4 \times 4$ system. With the current FEC scheme defined in the IEEE 802.11n standard, a $4 \times 4$ system requires twice amount of power in ADCs as a $2 \times 2$ system when receiving the same amount of information with the same power. However, using opportunistic error correction in a $4 \times 4$ system costs only around 1.4 times amount of energy in ADCs comparing to a $2 \times 2$ system.
\end{abstract}

\section{INTRODUCTION}

Multiple-Input Multiple-Output (MIMO) technology has attracted a lot of attention in wireless communications, due to its high data rate without additional bandwidth or transmitted power [1]. Combining Orthogonal Frequency Division Multiplexing (OFDM) with MIMO enables easy implementation of MIMO systems in wireless channels. OFDM has a high Peakto-Average Power Ratio (PAPR), therefore it needs Analog-toDigital Converter (ADC) with a high dynamic range, especially in MIMO-OFDM systems.

In this paper, we focus on a $\mathrm{M} \times \mathrm{M}$ MIMO-OFDM system with maximum multiplexing gain. At the transmitter, we assume that each antenna transmits one data stream and has unity transmission power. At the receiver, the signal arriving at each antenna is the combination of the signals from all the transmit antennas. So, the dynamic range of the ADCs is proportional to the number of antennas M. In wireless LAN systems, the ADC can consume up to $50 \%$ of the total baseband power budget [2]. Low power-consumption in battery-powered digital MIMO receivers is a highly desired feature. Therefore, we propose a novel scheme to reduce the power consumption of the ADC by combining a resolution adaptive ADC architecture with opportunistic error correction.

Fixed high-resolution ADCs are applied in current WLAN MIMO systems. The high-resolution ADCs are employed for worst-case channel conditions. However, worst-case channel conditions do not always happen. Therefore, we propose to use resolution adaptive ADCs whose resolution is adapted for each channel instead of being fixed for worst-case conditions. Correspondingly, the power consumption in ADCs is reduced.

This work was supported by the Dutch Ministry of Economic Affairs under the IOP - SenterNovem Program.
A further resolution reduction of the ADC can be achieved by using a novel error correction scheme to be integrated into the physical layer. In the IEEE 802.11n standard [3], the Forward Error Correction (FEC) layer encodes source data across all transmit antennas and the full transmission band, which means that each part of those $\mathrm{M}$ parallel channels (after inverting the effect of the MIMO channel) is considered equally important. In such case, the resolution of ADCs is determined by the sub-band with the lowest energy of the $\mathrm{M}$ channels. Take Figure 1 as an example, the lowest energy of the 4 parallel channels is around $-32 \mathrm{~dB}$. From this figure, we can see that the deep fading does not happen everywhere and only happens in the frequency band of 7 9 MHz. By discarding this $2 \mathrm{MHz}$ sub-band, the lowest energy of this channel vector is reduced to around $-20 \mathrm{~dB}$. If we put the 4 channels together and sort them by energy, its lowest energy is increased to around $-13 \mathrm{~dB}$ by also discarding $10 \%$ of bandwidth.

As just mentioned, the FEC scheme defined in the IEEE 802.11n standard does not allow us to discard part of a channel with deep fading. However, this can be achieved by using fountain codes. Fountain codes can reconstruct the source file by collecting enough packets. It does not matter which packet is received. We only need to receive a certain number of packets. Therefore, we propose to transmit a fountain-encoded packet over a sub-carrier per antenna. Multiple packets are transmitted simultaneously, using frequency division multiplexing and space division multiplexing. The receiver discards the sub-carriers with deep fading and recovers the source data by only collecting the well-received packets from the highenergy sub-carriers. With this approach, the quantization of the ADCs can be coarse.

The outline of this paper is as follows. We propose two techniques to reduce power consumption of ADCs: fountain codes and resolution adaptive ADCs. First, fountain codes are discussed which is followed by resolution adaptive ADCs for MIMO-OFDM systems. In Section IV, system models will be described. Finally, the simulation results will be analyzed, which compare the conventional 802.11n system with our approach. The paper ends with conclusions.

\section{Fountain Codes}

Opportunistic error correction is based on fountain codes. A fountain code has a similar property to a fountain of water: when you fill a cup from the fountain, you do not care about which drops of water fall in, but only want that your cup fills enough to quench your thirst [4]. 


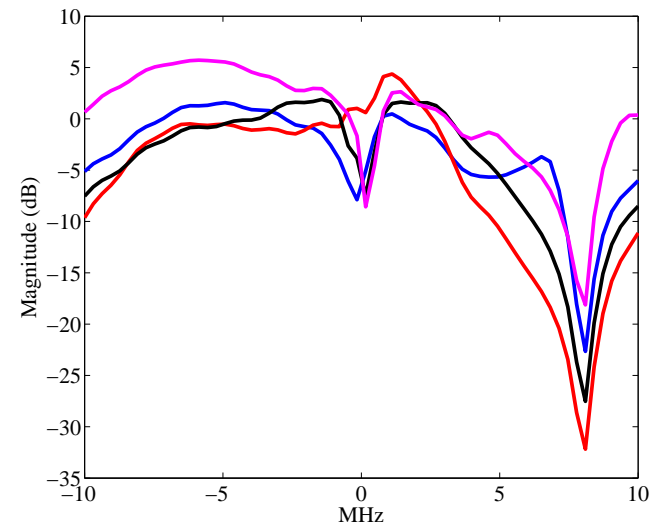

Fig. 1. Example of the baseband transfer function of a 4-by-4 Channel model A after inverting the effect of the MIMO channel.

With a fountain code, the transmitter can generate a potentially infinite supply of fountain-encoded packets and the receiver can reconstruct the original file by collecting enough fountain-encoded packets. The number of fountain-encoded packets $N$ required at the receiver is slightly larger than the number of source packets $K$ [5]:

$$
N=(1+\varepsilon) K
$$

where $\varepsilon$ is the percentage of extra packets and is called the overhead.

The message-passing algorithm has been used to reconstruct source data from fountain-encoded packets due to its linear computation cost [5]. However, it requires a large $\varepsilon$ for small block size. For example, the practical overhead of LT codes ${ }^{1}$ [7] is $14 \%$ when $K=2000$, which limits its application in practical systems [8]. In [9], we have shown that the overhead is reduced to $3 \%$ by using message-passing with Gaussian Elimination to decode LT codes for $K \geq 500$.

Fountain codes are specially designed for erasure channels. A wireless channel is not an erasure channel but a noisy fading channel. In order to apply fountain codes in wireless channels, error correction codes need to be utilized to make wireless channel behave like an erasure channel. In this paper, Low-Density Parity-Check (LDPC) codes together with Cyclic Redundancy Check (CRC) are used to convert the channel.

The opportunistic error correction scheme is performed as follows:

- At the transmitter, a fountain-encoded packet is created. Then the CRC is added. Finally, the packet is encoded by the LDPC code.

- At the receiver, each packet is first LDPC decoded if its energy is above or equal to a threshold (corresponding to $\mathrm{BER} \leq 10^{-5}$ ). The received packet is discarded if its energy is below the threshold. If the LDPC decoding fails, the received packet is discarded as well. If the

${ }^{1}$ LT code is a kind of fountain codes. In this paper, we use LT codes in our proposed scheme which is compatible with any fountain codes (e.g. Raptor codes [6]).
LDPC decoding succeeds, the CRC is used to identify any undetected errors from LDPC codes. If the CRC decoder detects an error, the receiver assumes that the whole packet has been lost. Once the receiver collects $N$ surviving fountain-encoded packets, it starts to recover the original data.

\section{RESOlution AdAPTIVE ADCs FOR MIMO-OFDM SYSTEM}

In MIMO-OFDM systems, signal detection is performed in the frequency domain after inverting the effect of the channel. It is not beforehand clear how many ADC bits are required for proper detection, because quantization happens in the time domain and before the effect of the MIMO channel is inverted. In this section, we derive a relation between the quantization noise in the time domain and the frequency domain for MIMOOFDM systems. Afterwards, we present a scheme to design optimum low-resolution ADCs for MIMO-OFDM systems.

Notation: bold represents vector and matrix, lower case represents the signal in the time domain and upper case represents the signal in the frequency domain.

\section{A. Minimum Number of Quantization Levels}

Because the quantization noise is dependent on the signal, we first analyze the statistical characteristics of the ADC input $\mathbf{r}_{n}$. We assume that the quantization noise is dominant, so other noise (e.g. channel noise) is ignored in this paper. In such case, a $\mathrm{M} \times \mathrm{M}$ channel output at the $n_{\mathrm{th}}$ moment $\mathbf{r}_{n}$ can be written as:

$$
\mathbf{r}_{n}=\sum_{l=0}^{L-1} \mathbf{h}_{l} \mathbf{x}_{n-l}
$$

where $\mathbf{x}=\left[\mathrm{x}^{(1)}, \cdots, \mathrm{x}^{(m)}, \cdots, \mathrm{x}^{(\mathrm{M})}\right]$ is the transmitted vector, $\mathbf{h}_{l}$ is the $\mathrm{M} \times \mathrm{M}$ channel matrix at the $l_{\mathrm{th}}$-path and $L$ is the number of channel paths. From [10], we know that $\mathrm{x}^{(m)}$ can be modeled as a normal-distributed random variable with zero mean and a variance of 1 . The elements in $\mathbf{x}$ are mutual independent, so it has zero mean and a unity variance matrix. In addition, $\mathbf{x}_{n}$ and $\mathbf{x}_{n^{\prime}}$ are mutual independent, if $n \neq n^{\prime}$.

As each element in $\mathbf{r}_{n}=\left[\mathrm{r}_{n}^{(1)}, \cdots, \mathrm{r}_{n}^{(m)}, \cdots, \mathrm{r}_{n}^{(\mathrm{M})}\right]$ is quantized separately, we analyze the statistical characteristics of $\mathbf{r}_{n}^{(m)}$ instead of $\mathbf{r}_{n} . \mathbf{r}_{n}^{(m)}$ can be expressed as:

$$
\mathrm{r}_{n}^{(m)}=\sum_{l=0}^{L-1} \sum_{s=0}^{\mathrm{M}-1} h_{l}^{(m, s)} x_{n-l}^{(s)}
$$

According to the Central Limit Theorem, the sum of a sequence of independent, identically distributed random variables tends to be Gaussian-distributed, so the probability density function of $\mathrm{r}_{n}^{(m)}$ can be derived as:

$$
f\left(\mathrm{r}_{n}^{(m)}\right) \approx \frac{1}{\pi \sigma_{\mathrm{r}_{n}^{(m)}}^{2}} \exp \left(-\frac{\left|\mathrm{r}_{n}^{(m)}\right|^{2}}{\sigma_{\mathrm{r}_{n}^{(m)}}^{2}}\right)
$$

where $\sigma_{\mathrm{r}_{n}^{(m)}}^{2}=\sum_{l=0}^{L-1} \sum_{s=0}^{\mathrm{M}-1}\left|h_{l}^{(m, s)}\right|^{2}$. For simplicity, we assume that $\sum_{l=0}^{L-1}\left|h_{l}^{(m, s)}\right|^{2}=\sum_{l=0}^{L-1}\left|h_{l}^{\left(m, s^{\prime}\right)}\right|^{2}=1\left(s \neq s^{\prime}\right)$, 
so $\mathrm{r}_{n}^{(m)} \sim \mathcal{C N}(0, \mathrm{M})$. However, the discussion below holds more generally.

The ADC output $\mathrm{y}_{n}^{(m)}$ can be expressed as:

$$
\mathrm{y}_{n}^{(m)}=\mathcal{Q}\left(\mathrm{r}_{n}^{(m)}\right)=\sum_{l=0}^{L-1} \sum_{s=0}^{\mathrm{M}-1} h_{l}^{(m, s)} x_{n-l}^{(s)}+\mathrm{n}_{n}^{(m)}
$$

where $\mathrm{n}_{n}^{(m)}$ is the quantization noise in the time domain at the $m_{\mathrm{th}}$ antenna. With a uniform quantizer, $\mathrm{n}_{n}^{(m)}$ is uniformly distributed with zero mean and a variance of $\frac{\Delta^{2}}{6}$, where $\Delta$ is the uniform quantization step [10].

After OFDM demodulation, each sub-carrier can be considered as a narrow-band MIMO channel in the frequency domain:

$$
\mathbf{Y}_{k}=\mathbf{H}_{k} \mathbf{X}_{k}+\mathbf{N}_{k}
$$

where $\mathbf{X}_{k}=\left[\mathrm{X}_{k}^{(1)}, \cdots, \mathrm{X}_{k}^{(\mathrm{m})}, \cdots, \mathrm{X}_{k}^{(\mathrm{M})}\right]$ is the transmitted vector at the $k_{\text {th }}$ sub-carrier and $\mathbf{N}_{k}=\left[\mathrm{N}_{k}^{(1)}, \cdots, \mathrm{N}_{k}^{(\mathrm{m})}, \cdots, \mathrm{N}_{k}^{(\mathrm{M})}\right]$ is the quantization noise in the frequency domain:

$$
\mathbf{N}_{k}=\frac{1}{\sqrt{\mathrm{N}}} \sum_{n} \mathbf{n}_{n} e^{-j \frac{2 \pi}{\mathrm{N}} n k}
$$

where $\mathrm{N}$ is the number of sub-carriers. The Central Limit Theorem shows that $\mathrm{N}_{k}^{(m)}$ is Gaussian-distributed with zero mean and a variance of $\frac{\Delta^{2}}{6}$ [10]. $\mathbf{H}_{k}$ is the fading matrix at the $k_{\text {th }}$ sub-carrier defined by:

$$
\mathbf{H}_{k}=\sum_{l=0}^{L-1} \mathbf{h}_{l} e^{-j \frac{2 \pi}{N} l k}
$$

In order to detect the transmitted symbol from each transmitted antenna, we need to invert the effect of the MIMO channel. In this paper, the zero-forcing algorithm is used to do the inversion:

$$
\hat{\mathbf{X}}_{k}=\mathbf{H}_{k}^{-1} \mathbf{Y}_{k}=\mathbf{X}_{k}+\mathbf{H}_{k}^{-1} \mathbf{N}_{k}
$$

Thus, for the symbol $\mathrm{X}_{k}^{(m)}$ from the $m_{\mathrm{th}}$ antenna at the $k_{\mathrm{th}}$ sub-carrier, its Signal-to-Noise Ratio (SNR) can be derived as:

$$
\mathrm{SNR}_{k}^{(m)}=\left(\frac{\Delta^{2}}{6} \sum_{s=0}^{M-1}\left|\mathbf{H}_{k}^{-1}{ }^{(m, s)}\right|^{2}\right)^{-1}
$$

The above equation shows the quantization effects in MIMO-OFDM system. To communicate reliably, error correction coding is used to mitigate the quantization effect. Different coding has different SNR threshold to achieve a BER at a certain order or lower (e.g. $10^{-4}$ ). So, the quantization step $\triangle$ can be derived once an error correction code is chosen and the channel is estimated. If clipping is allowed, the number of quantization levels $N_{q}$ can be determined by [10]:

$$
N_{q}=2\left\lceil\frac{\mathrm{C}}{\triangle}\right\rceil
$$

where $\mathrm{C}=3 \sigma_{\mathrm{r}_{n}^{(m)}}=3 \sqrt{\mathrm{M}}$. Here, we can see that the dynamic range of ADCs is proportional to $\mathrm{M}$, so does $N_{q}$.
From Equation 10 and 11, we can see that $N_{q}$ is determined by $\mathrm{M}, \triangle$ and the channel. Once the MIMO system is chosen and the channel is fixed, $N_{q}$ depends on $\Delta$. In such case, $\Delta$ depends not only on the used error correction code but also on how an encoded packet is transmitted.

\section{B. Power Consumption}

In general, the power consumption of ADCs is proportional to the number of quantization levels $N_{q}$. The power consumption of the ADC implemented by CMOS scales linearly with $N_{q}$ [2]. Thus, we can write the ADC's power consumption P as:

$$
\mathrm{P}=\sum_{i=0}^{M_{c}-1} \sum_{m=0}^{\mathrm{M}-1} \alpha_{i} N_{q_{i}}^{(m)} \mathcal{M}
$$

where $M_{c}$ is the number of channel realizations, $\alpha_{i}$ is the percentage of the $i$-th channel realization where useful information is transmitted, $N_{q i}^{(m)}$ is the number of quantization levels used in the $i$-th channel realization and at the $m$-th antenna, and $\mathcal{M}$ is the number of samples per MAC frame.

Once an error correction code is chosen, Equation 10, 11 and 12 tell us that the power consumption of ADCs depends on the sub-carrier with the lowest energy. By transmitting one fountain-encoded packet over a sub-carrier per antenna, the power consumption of ADCs can be reduced by discarding packets from the sub-carriers with deep fading. For example, assume that a fountain-encoded packet is transmitted over a 4-by-4 channel as Figure 1. Method I is to transmit it across all the antennas and all the sub-carriers as defined in [3] and Method II is to transmit it over one sub-carrier per antenna. Suppose the fountain-encoded packet can be received correctly when its SNR $\geq 12 \mathrm{~dB}$. Figure 2 shows that Method I needs 778 levels but Method II only requires 62 levels in ADC by discarding $18 \%$ of the sub-carriers. However, discarding packets over the low-energy sub-carriers means increasing the total number of transmitted packets which might increase the total power consumption in ADCs. Therefore, there is a tradeoff in the power consumption between the number of lost sub-carriers and the total number of transmitted packets which will be analyzed in the following section.

\section{System Model}

The opportunistic error correction scheme is based on fountain codes and resolution adaptive ADCs which have been explained in the above sections. This novel method can be applied in MIMO-OFDM systems. In this paper, we take the IEEE 802.11n system as an example of MIMO-OFDM systems.

The current IEEE 802.11n standard is based on Rate Compatible Punctured Codes (RCPC). RCPC has good performance for random bit errors but not for burst bit errors, so interleaving is applied to reduce burst errors. Each RCPCencoded packet is transmitted based on Method I. Although this solution works good in practice, it is not optimal from a power consumption point of view: 


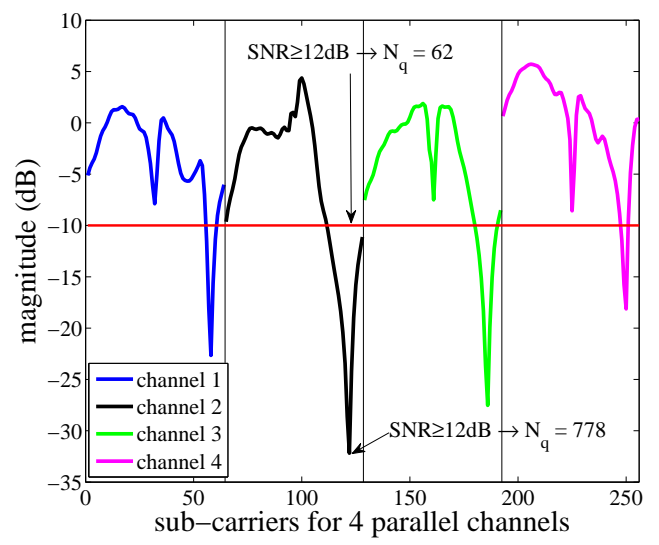

Fig. 2. The difference in the number of ADC levels $N_{q}$ between transmission Method I and transmission Method II.

- Packets encountered by a "bad" channel are still processed by the receiver.

- Fixed high-resolution ADCs are used in all antennas.

Those problems can be solved by using the opportunistic error correction scheme, as shown in Figure 3. The key idea is to transmit fountain-encoded packets based on Method II and generate additional packets by the fountain encoder. At the transmitter, the source file is first divided into a stream of source packets which are encoded by a LT code (with parameter $c=0.03, \sigma=0.3)$. Then, each fountain-encoded packet is added by a 7-bit CRC checksum and encoded by a $(175,255)$ LDPC code. Afterwards, it is transmitted over a sub-carrier of a transmitted antenna.

At the receiver, the resolution of ADCs is adapted into the minimum with the estimated channel knowledge and the number of lost sub-carriers. After OFDM demodulation and inverting the effect of the MIMO channel, the receiver can only process the packets from high-energy sub-carriers (i.e. SNR $\geq$ threshold) by checking its SNR defined in Equation 10. The received packet survives if it has successful LDPC decoding and $\mathrm{CRC}$ checking. Once the receiver has got enough packets, it reconstructs the original file by fountain decoding.

As discussed in Section III, discarding more sub-carriers does not mean less power consumption in ADCs. Assume that the receiver needs to collect 1000 fountain-encoded packets and the received packet will be discarded if its SNR is less than $12 \mathrm{~dB}$. With a HT (i.e. High Throughput) mode defined in [3], 52 sub-carriers are used to transmit fountain-encoded packets. As the quantization noise is the dominant noise, resolution adaptive ADCs are designed in such way that $N_{b}^{2}$ sub-carriers have a SNR lower than $12 \mathrm{~dB}$. Figure 4 shows the tradeoff between the power consumption in ADCs and $N_{b}$ with perfect channel knowledge. As we can see, a $4 \times 4$ system consumes more power in ADCs than a $2 \times 2$ system to receive the same amount of fountain-encoded packets. Their difference is reduced when $N_{b}>0$.

\footnotetext{
${ }^{2} N_{b}$ : the number of lost sub-carriers
}

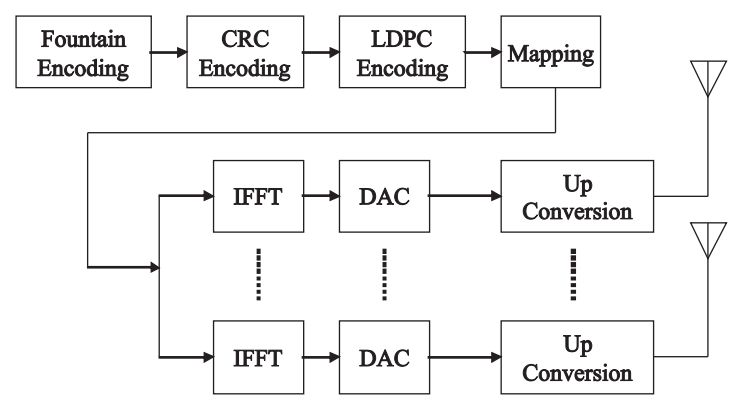

(a) Transmitter

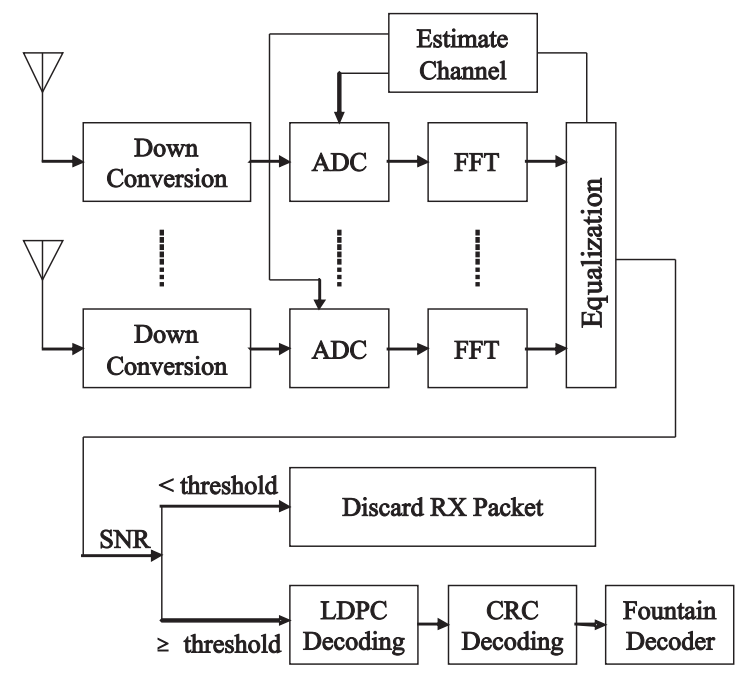

(b) Receiver

Fig. 3. Proposed IEEE 802.11n transmitter (top) and receiver (bottom).

The power consumption of ADCs in both systems first decreases then increases when $N_{b}$ becomes larger. The $2 \times 2$ system consumes the minimum power in ADCs with 17 lost sub-carriers while the $4 \times 4$ system needs to discard 19 subcarriers. Before arriving the minimum point, the decreasing speed becomes slower when $N_{b}$ increases. Comparing to $N_{b}=0$, the power consumption in the $2 \times 2$ system is reduced by around $62 \%$ when $N_{b}=5,69 \%$ when $N_{b}=10$ and $71 \%$ when $N_{b}=17$. Similar valued holds for the $4 \times 4$ system, whose power consumption in ADCs is decreased by around $73 \%$ with 5 lost sub-carriers, $78 \%$ with 10 lost sub-carriers and $80 \%$ with 19 sub-carriers lost comparing to the case of no lost sub-carrier. Obviously, the power saving in ADCs is mainly contributed by discarding 5 lowest-energy sub-carriers. In addition, the $4 \times 4$ system has more power saving in ADCs than the $2 \times 2$ system given a $N_{b}$.

\section{PERFormance ANALYSis}

In this section, we analyze the performance of opportunistic error correction scheme by comparing the following scenarios. Scenario I is a RCPC with interleaving from the IEEE 802.11n standard with $R_{\mathrm{I}}=0.5$. Fixed-resolution ADCs are used in Scenario I. As the standard allows $10 \%$ packet loss, the resolution of ADCs in Scenario I is designed for $90 \%$ channel realizations. In Scenario II, we replace fixed-resolution ADCs 


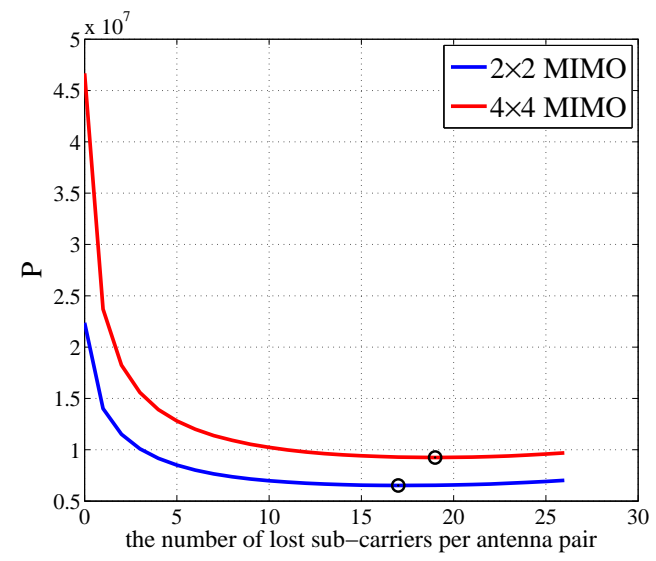

Fig. 4. Power consumption defined in Equation 12 versus the number of lost sub-carriers for receiving 1000 fountain-encoded packets.

in Scenario I by resolution adaptive ADCs. Scenario III is the opportunistic error correction scheme based on fountain codes and resolution adaptive ADCs. In Scenario III, the code rate of LT code $R_{\mathrm{LT}}$ is around 0.97 and the code rate of LDPC plus CRC $R_{\mathrm{LDPC}-\mathrm{CRC}}$ is around 0.66 , so its total code rate $R_{\mathrm{III}}$ is around 0.64 . To have the same effective throughput, Scenario III is allowed to discard around $30 \%$ (i.e. $0.5 \times 0.9 / 0.64$ ) of data sub-carriers. In addition, QAM-16 is the mapping scheme and 52 sub-carriers are used to transmit data as defined in [3].

Figure 5 shows the simulation results for a $2 \times 2$ system and a $4 \times 4$ system. In both systems, Scenario I costs the most power in ADCs comparing to Scenario II and III. Scenario III saves around $65 \%$ ADCs' power in a $2 \times 2$ system $(79 \%$ for a $4 \times 4$ system) comparing to Scenario II, which consumes around $49 \%$ of the power in ADCs with respect to Scenario I in a $2 \times 2$ system ( $47 \%$ for a $4 \times 4$ system). As we can see, Scenario III in a $4 \times 4$ system has more energy saving than in a $2 \times 2$ system in comparison with Scenario I and II.

In addition, receiving the same amount of information in a $2 \times 2$ system consumes more power in ADCs than in a $4 \times 4$ system. With RCPC, the power consumption in ADCs using a $4 \times 4$ system is around twice as much as the case of using a $2 \times 2$ system no matter whether using fixed-resolution ADCs or resolution adaptive ADCs. However, this does not happen in the opportunistic error correction scheme. In Scenario III, receiving the same amount of information using a $4 \times 4$ system consumes around 1.4 times amount of power in ADCs as a $2 \times$ 2 system. Furthermore, the receiver does not need to process all the received packets but only process the well-received packets in Scenario III, which does not apply in Scenario I and II.

\section{Conclusions}

In this paper, we propose an energy-efficient scheme based on fountain codes and resolution adaptive ADCs for MIMOOFDM systems to save power consumption in ADCs. The $\mathrm{ADCs}$ in a receiver can consume up to $50 \%$ of the total baseband energy, so the need to lower its power consumption is

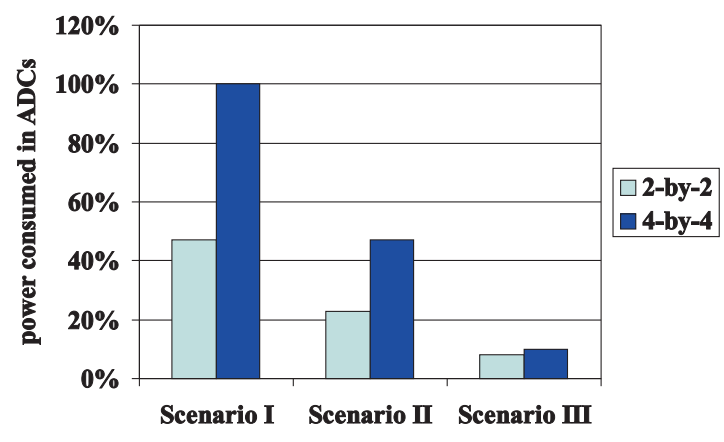

Fig. 5. Comparison in power consumption between Scenario I, II and III for a $2 \times 2$ system and a $4 \times 4$ system. Scenario I: RCPC with fixedresolution ADCs, Scenario II: RCPC with resolution adaptive ADCs, Scenario III: opportunistic error correction (i.e. fountain codes with resolution adaptive ADCs). The power consumption of Scenario I in a $4 \times 4$ system is normalized to 1 .

clear. The resolution adaptive ADCs saves around $49 \%$ power in a $2 \times 2$ system and $47 \%$ power in a $4 \times 4$ system comparing to fixed-resolution ADCs. Fountain codes together with LDPC plus CRC codes allow another $34 \%$ energy saving in a $2 \times 2$ system and $43 \%$ in a $4 \times 4$ system comparing to RCPC. In total, our proposed approach gives a power saving in ADCs of around $83 \%$ in a $2 \times 2$ system and a saving of around $90 \%$ in a $4 \times 4$ system.

Furthermore, the power consumption in ADCs is proportional to $\mathrm{M}$ by receiving the same amount of information and with the same transmission power. With RCPC, a $4 \times 4$ system costs about twice the power in ADCs comparing to a $2 \times 2$ system. However, fountain codes allow a $4 \times 4$ system to consume around 1.4 times of the amount of power in ADCs with respect to a $2 \times 2$ system.

\section{REFERENCES}

[1] D. Tse and P. Viswanath, Fundamentals of Wireless Communication. New York, NY, USA: Cambridge University Press, 2005.

[2] J. Thomson, et al., "An integrated 802.11 a baseband and MAC processor," in 2002 IEEE International Solid-State Circuits Conference (ISSCC), vol. 2, 2002.

[3] IEEE, "Draft Standards for Wireless LAN Medium Access Control (MAC) and Physical Layer (PHY) Specifications, Enhancements for Higher Throughput (IEEE 802.11n Standard, Part 11)," Jan, 2007.

[4] M. Mitzenmacher, "Digital fountains: A survey and look forward," in IEEE Information Theory Workshop, 2004, pp. 271-276.

[5] D.J.C. MacKay, Information Theory, Interference, and Learning Algorithms. Cambridge University Press, 2003.

[6] A. Shokrollahi, "Raptor codes," IEEE Transactions on Information Theory, no. 6, pp. 2551-2567.

[7] M. Luby, "LT Codes," Proceedings of the 43rd Annual IEEE Symposium on Foundations of Computer Science, pp. 271-282, 2002.

[8] X. Shao, R. Schiphorst, and C. H. Slump, "Opportunistic Error Correction for WLAN Applications," Proceedings of the 4th IEEE International Conference on Wireless Communications, Networking and Mobile Computing, 2008.

[9] X. Shao and R. Schiphorst and C. H. Slump, "An Opportunistic Error Correction Layer for OFDM Systems," EURASIP Journal on Wireless Communications and Networking, 2009.

[10] X. Shao and C. H. Slump, "Quantization Effects in OFDM Systems," in Proceedings of 29th Symposisum on Information Theory in the Benelux, Leuven, May 2008. 